\title{
Gene Expression Profiling in Rice Infected with Rice Blast Fungus using SAGE
}

\author{
Sang Gon Kim ${ }^{1,4, \uparrow}$, Sun Tae Kim ${ }^{1, \uparrow}$, Sung-Kun Kim ${ }^{4}$ and Kyu Young Kang ${ }^{1,2,3 *}$ \\ ${ }^{1}$ Environmental Biotechnology National Core Research Center, ${ }^{2}$ Division of Applied Life Science (BK21 Program), \\ ${ }^{3}$ Plant Molecular Biology and Biotechnology Research Center, Gyeongsang National University, Jinju 660-701, Korea \\ ${ }^{4}$ Department of Chemistry and Biochemistry and Institute of Biomedical Studies, Baylor University, Texas 76798-7348, USA \\ (Received on July 1, 2008; Accepted on November 5, 2008)
}

\begin{abstract}
Rice blast disease, caused by the pathogenic fungus Magnaporthe grisea, is a serious issue in rice (Oryza sativa L.) growing regions of the world. Transcript profiling in rice inoculated with the fungus has been investigated using the transcriptomics technology, serial analysis of gene expression (SAGE). Short sequence tags containing sufficient information which are ten base-pairs representing the unique transcripts were identified by SAGE technology. We identified a total of 910 tag sequences via the GenBank database, and the resulting genes were shown to be up-regulated in all functional categories under the fungal biotic stress. Compared to the compatible interaction, the stress and defense genes in the incompatible interaction appear to be more up-regulated. Particularly, thaumatin-like gene (TLP) was investigated in determining the gene and protein expression level utilizing Northern and Western blotting analyses, resulting in an increase in both the gene and the protein expression level which arose earlier in the incompatible interaction than in the compatible interaction.
\end{abstract}

Keywords : abiotic stress, Magnaporthe grisea, Oryza sativa, pathogenesis-related gene, rice blast, SAGE

One of the most devastating diseases in rice, called rice blast, is caused by Magnaporthe grisea (Hamer and Talbot, 1998; Kim et al., 2001). The rice blast disease is a very serious and recurrent issue in rice-growing regions of the world (Talbot, 2003). The infection usually occurs on rice plant leaves where the fungal spores of $M$. grisea land and attach themselves using the fungal unique adhesive structure called an appressorium (Hamer et al., 1988) to penetrate rice leaf surface by building high turgor pressure (De Jong et al., 1997). Every year, the rice blast infection has the potential to affect the amount of harvested rice by which about sixty million people can be fed (Zeifler et al., 1994), generating significant economical and humanitarian issues in rice-growing areas. It has also been reported that some

\footnotetext{
*Corresponding author.

Phone) +82-55-751-5468, FAX) +82-55-757-0178

E-mail)kykang@gnu.ac.kr

These authors contributed equally to this work.
}

strains of the fungal $M$. grisea not only kill rice, but also attack other major cereals and grasses (Valent and Chumley, 1991).

In an effort to remedy this agricultural crisis, finding the genes that are able to defend against the fungal biotic stress would be of great value. It is an obvious interest to determine which genes would be involved in the regulation and with what gene expression level in response to M. grisea infection. Transcriptomics can be used to determine the gene expression level of the messenger RNA (mRNA) in a given cell population. Transcriptomics is the study of the transcriptome, which is the set of all mRNA or transcripts produced in a population of cells. The transcriptome varies under stress conditions because all mRNA transcripts in a cell are a reflection of the genes that are being actively expressed under any stress conditions. Two technologies can be useful tools for transcriptomics. One of the technologies is microarray analysis, which utilizes labeled cDNAs hybridized to an array of DNA elements as probes affixed to a solid support. Using microarray analysis, high densities are achievable and enable the measurement of over 10,000 genes. Microarray is one of the popular technologies that contain information derived from hundreds or even thousands of samples. As another approach to transcriptomics, serial analysis of gene expression (SAGE) can be used, which has been developed by Velculescu et al. (1995). SAGE technology is also a powerful tool that allows the analysis of overall gene patterns. The level of gene expression of an organism in a variety of normal, developmental, and disease states can be quantified by SAGE technology in the same way as microarray technology. The principle of the SAGE methodology is to use a short sequence tag containing sufficient information to uniquely identify a transcript. The size of the short sequence tag is normally between ten and fourteen base pairs (bp) that will likely be chosen from a unique position within each transcript. The tags will then be linked together to form long serial tags that have multiple pieces of transcript information. After cloning the long serial tags, we can quantify the number of tags, which are demonstrating the gene expression levels of the corresponding transcripts. SAGE tags, therefore, are used not only for gene identification, but also for measuring 
the relative abundance of their cognate transcripts within the mRNA population based on the number of occurrences of a given SAGE tag.

In this study, we prepared three different kinds of rice leaves: leaves that grew under no stress conditions (as a control), leaves that conferred resistance to $M$. grisea infection (called an incompatible interaction) and leaves that displayed susceptibility to the fungal infection (called a compatible interaction). SAGE technology was applied to the prepared leaves in an effort to find the genes upregulated in response to the $M$. grisea infection. We analyzed the types of genes by grouping them into specific categories, permitting us to identify the resistant genes. Northern and Western blotting analyses for a gene of interest were carried out for detailed information.

\section{Materials and Methods}

Plant materials. Rice (Oryza sativa) seedlings with 4-5 leaf stages grown under natural light in a greenhouse at a temperature of $28{ }^{\circ} \mathrm{C}$ were used for inoculation of whole plants with blast fungus $M$. grisea. $M$. grisea were sprayed onto the rice leaves as described previously (Kim et al., 2004a). The inoculated plants were kept in a humidity chamber at $28{ }^{\circ} \mathrm{C}$ and harvested at defined time periods.

Restriction endonuclease. The restriction endonuclease Sau3A1 (restriction recognition site: /GATC; the slash (/) represents the cleavage site) was used as an anchor. The type IIs restriction enzyme (also known as a tagging enzyme) Fok1 cleaves the sites 9-13 base pairs away from the recognition site (recognition site: GGATG (9-13)/).

Primer and Linker. Linkers should contain three important sequences: the appropriate anchoring enzyme overhang, a recognition site for a type IIs restriction enzyme, and a priming site for PCR amplification (Madden et al., 2000). Two SAGE linkers attached with biotinylated oligos (20 dT) (Integrated DNA Technologies) were used. SAGE linker 1 was formed through hybridization of oligonucleotides 1A (forward direction): 5'-TTTGCCAGGTCACTCTTCTCGGATACTCGTCACACAGGAT-3', and 1B (reverse direction): 5'-GATCATCCTGTGTGACGAGTATCCGAGAAGAGTGACCTGGC-3'. SAGE linker 2 was obtained by hybridizing oligonucleotide $2 \mathrm{~A}$ (forward direction): 5'TTTGCTCTGGACCTCTTGCTCGTCTAATGACTCTT GGAT-3' and 2B (reverse direction): 5'-GATCATCCAAGAGTCATTAGACGAGCAAGAGGTCCAGAGC-3'.

Oligonucleotides $1 \mathrm{~B}$ and $2 \mathrm{~B}$ included two modifications; 5'-end phosphorylation and 3'-end C7 amino modification. These were performed for the immobilization of the desired oligonucleotide on the beads. Oligonucleotides were purified from $12 \%$ native polyacrylamide gel. The sequence of cognate PCR primers for linkers 1 and 2 were 5'-GCCAGGTCACTCTTCTCGGA-3' and 5'-GCTCTGGACCTCTTGCTCGT-3', respectively.

RNA isolation and cDNA synthesis. Total RNA was extracted from rice leaves $24 \mathrm{~h}$ after inoculation with $M$. grisea by an SDS-phenol extraction protocol (Datson et al., 1999; Munasinghe et al., 2001; Yamamoto et al., 2001). mRNAs containing polyA were isolated using Oligotex-dT column (Qiagen Inc.) as recommended by the manufacturer. Five micrograms of the mRNA were used for doublestranded cDNA synthesis with a cDNA synthesis kit (Life Technologies Inc.). Protocols supplied with the kits were followed, except for the use of biotinylated oligo-dT.

SAGE procedure. SAGE procedure was performed according to the original protocol (Velculescu et al., 1995) with some modifications (Fig. 1). The cDNA was digested with the anchoring enzyme Sau3A1 (New England Biolabs) and captured with the 3 ' end cDNA through binding to streptavidin-coated magnetic beads (New England Biolabs). The streptavidin-bound cDNA was divided into two fractions. Linkers A and B were ligated to the captured 3' end cDNA, and SAGE tags adjacent to the linkers were released from beads by Fok1 (New England Biolabs) digestion. The released cDNA tags were blunt-ended, and the two fractions were then ligated to each other by using T4 DNA ligase $(5 \mathrm{U} / \mu \mathrm{L})$ purchased from Gibco BRL. Ditags were amplified by PCR using ExTaq DNA polymerase purchased from TaKaRa Co., and specific primers (5'GCCAGGTCACTCTTCTCGGA-3' and 5'-GCTCTGGACCTCTTGCTCGT-3') were used for the biotinylated linker. The sample derived from PCR was loaded on ten lanes of $12 \%$ polyacrylamide gel (Margulies et al., 2001). The region around $100 \mathrm{bp}$ was excised across all ten lanes of the gel; we then removed the polyacrylamide using SpinX columns (Costar Co.) (Angelastro et al., 2000). Amplified fragments (24-28 bp) were released from the linkers and purified again from polyacrylamide gels. The purified ditags were purified again using the affinity bead. Tag concatemers were obtained by ligation of purified ditags (Powell, 1998; Kenzelmann and Muhlemann, 1999). Before cloning to the pBluescript $\mathrm{SK}(+)$ vector (Stratagene), concatemers were size-fractionated (>300 bp). E. coli XL1 blue competent cells were transformed with the subcloned plasmids. Colonies on the Luria-Bertani medium containing $50 \mu \mathrm{L} / \mathrm{mL}$ of ampicillin were screened for the plasmids (van den Berg et al., 1999).

Sequencing and analysis of clones. PCR with vector- 


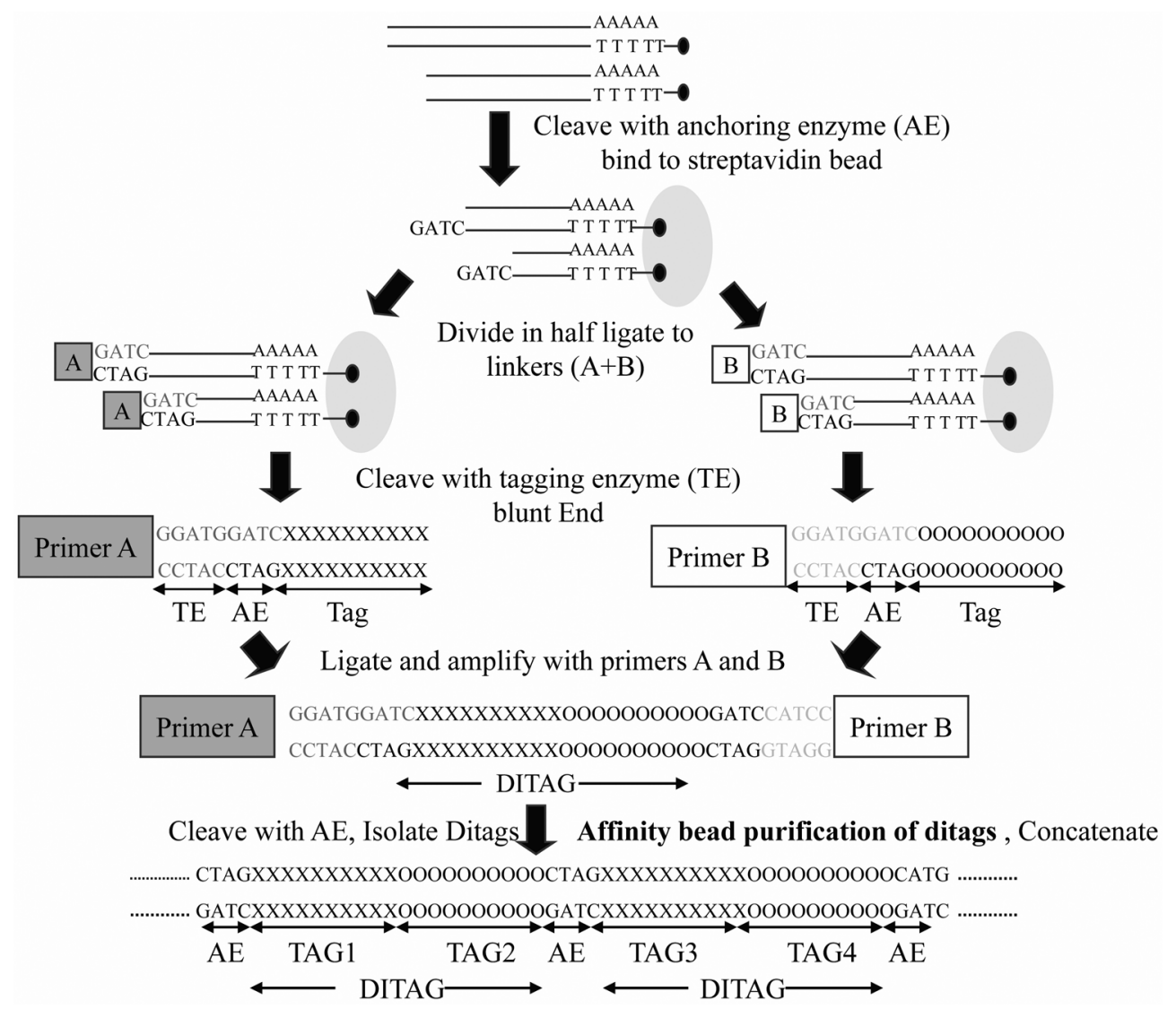

Fig. 1. Schematic diagram of SAGE protocol. The anchoring enzyme is Sau3A1 and the tagging enzyme is Fok1. Sequences colored gray represent primer-derived sequences, while black (X and $\mathrm{O}$ ) represents transcript-derived sequence, with $\mathrm{X}$ and $\mathrm{O}$ indicating nucleotides of different tags. AE and TE represent an anchor enzyme and a tagging enzyme, respectively.

specific primers was performed on individual bacterial colonies containing cloned concatemers in order to determine the insert length. PCR products with a size larger than $400 \mathrm{bp}$, which contain more than 12 tags, were selected for sequence analysis (Peters et al., 1999). Direct sequencing of PCR product was performed using a $377 \mathrm{ABI}$ automated sequencer with BigDye Primer Kit (ABI Inc.).

Northern blot analysis. The total mRNA $(20 \mu \mathrm{g})$ was denatured and separated by electrophoresis on a $1.5 \%(\mathrm{w} / \mathrm{v})$ agarose-formaldehyde gel and then transferred onto a nylon membrane (GeneScreen Plus, NEN, Boston). Filters were prehybridized for $30 \mathrm{~min}$ and incubated for 12-16 hrs at 65 ${ }^{\circ} \mathrm{C}$ in a Church's hybridization solution $\left(0.5 \mathrm{M} \mathrm{Na}_{2} \mathrm{HPO}_{4}\right.$, $\mathrm{pH} 7.2,1 \mathrm{mM}$ EDTA, $1 \%$ BSA, and 7\% SDS) (Church and Gilbert, 1984) with a partial thaumatin like protein (TLP) cDNA as a probe. The membranes were washed three times for 15 minutes with $2 \mathrm{X} \mathrm{SSC}$ containing $0.1 \% \mathrm{SDS}$ at $65^{\circ} \mathrm{C}$, and then three times with $0.2 \mathrm{X}$ SSC containing $0.1 \%$ SDS at $6^{\circ} \mathrm{C}$ for $15 \mathrm{~min}$. The blot was exposed to Kodak XAR-5 $\mathrm{X}$-ray film at $-70^{\circ} \mathrm{C}$. An equal sample amount $(20 \mu \mathrm{g})$ was loaded on the gel to confirm the presence of samples via the ethidium bromide staining method.

Antibody production and immunoblot analysis. The TLP antiserum was generated by immunizing a rabbit with the purified 6X His::TLP fusion protein. Specificity of the specific antibody was tested by probing the purified $6 \mathrm{X}$ His::TLP recombinant protein produced in E. coli. For immunoblot analysis, the protein sample was subjected to SDS-PAGE, and electrotransfered onto polyvinylidene fluoride (PVDF) membranes using an electrotransfer kit (Hoefer Scientific Instrument). The membrane was washed once with $\mathrm{H}_{2} \mathrm{O}$, blocked with $6 \%(\mathrm{w} / \mathrm{v})$ nonfat dried milk (Carnation, Glendale, CA) in TBS (20 mM Tris, $\mathrm{pH} 7.5$ and $150 \mathrm{mM} \mathrm{NaCl}$ ) containing $0.05 \%$ (v/v) Tween 20 (TBS-T) for 2-4 hrs at room temperature, and washed with TBS-T. The rabbit polyclonal anti-6X His::TLP (total: diluted $1: 5000$ with TBS-T containing $6 \%(\mathrm{w} / \mathrm{v})$ nonfat dried milk) antibody was then added, and the membrane was incubated for one hour at room temperature, washed three times with TBS-T, and treated with horseradish peroxidase-conjugated mouse anti-rabbit IgG (Sigma: diluted 1:5000) in TBS-T containing $6 \%(\mathrm{w} / \mathrm{v})$ nonfat dried milk for one hour at room 
temperature. Finally, after four washes with TBS-T, the protein bands were visualized using the enhanced chemiluminescence western blot detection system (ECL, Amersham Pharmacia Biotech).

\section{Results and Discussion}

To find the gene that confers resistance to the rice blast disease, we successfully prepared compatible and incompatible interactions of $M$. grisea and separately prepared leaves that grew under no stress conditions as a control (see the details in Materials and Methods). When we applied SAGE technology to each set of leaves, we modified the methods to improve the efficiency of PCR amplification and the formation of both ditags and concatemers. Because the efficiency of SAGE was limited by multiple gel purification steps by which the possibility of contamination was increased and the activity of the enzymes was reduced or inhibited, we modified the protocol using biotinylated-affinity bead purification for the 28 mer ditags (see Methods). We successfully formed ditags, confirmed by $12 \%$ native polyacrylamide gel (Fig. 2). We selected concatemers based on the DNA bands visualized with ethidium bromide on a
$1.0 \%$ agarose gel, and selected the bands that were bigger than $400 \mathrm{bp}$ in a way that obtains sufficient gene information at each DNA sequencing trial. Note that prior to the sequencing, subcloning was carried out using pBluesciprt SK (+). In total, more than fifty samples have been subcloned in order to determine the tag gene sequences from the compatible and incompatible interactions, along with the control. As a result, we acquired 531 tag sequence data for control, 1,150 tag sequence data for the incompatible interaction, and 1,064 tag sequence data for the compatible interaction. A total number of 2,745 tag sequence data have been acquired. We confirmed that all sequencing data contained the marker sequence GATC and two ten nucleotide bases in a ditag. In an effort to analyze the tag gene sequences, we input all resulting sequence data into the NCBI-Blast search engine. Although we were not able to identify all of the possible genes, we confirmed the identities of 910 genes; 176 gene identities for the control, 372 gene identities for the incompatible interaction, and 362 gene identities for the compatible interaction were determined (Supplementary Table). Through SAGE analysis, a total of 2,745 tags were collected; 910 (33\%) tags were identified, but 1,835 could not be identified from the Gen-

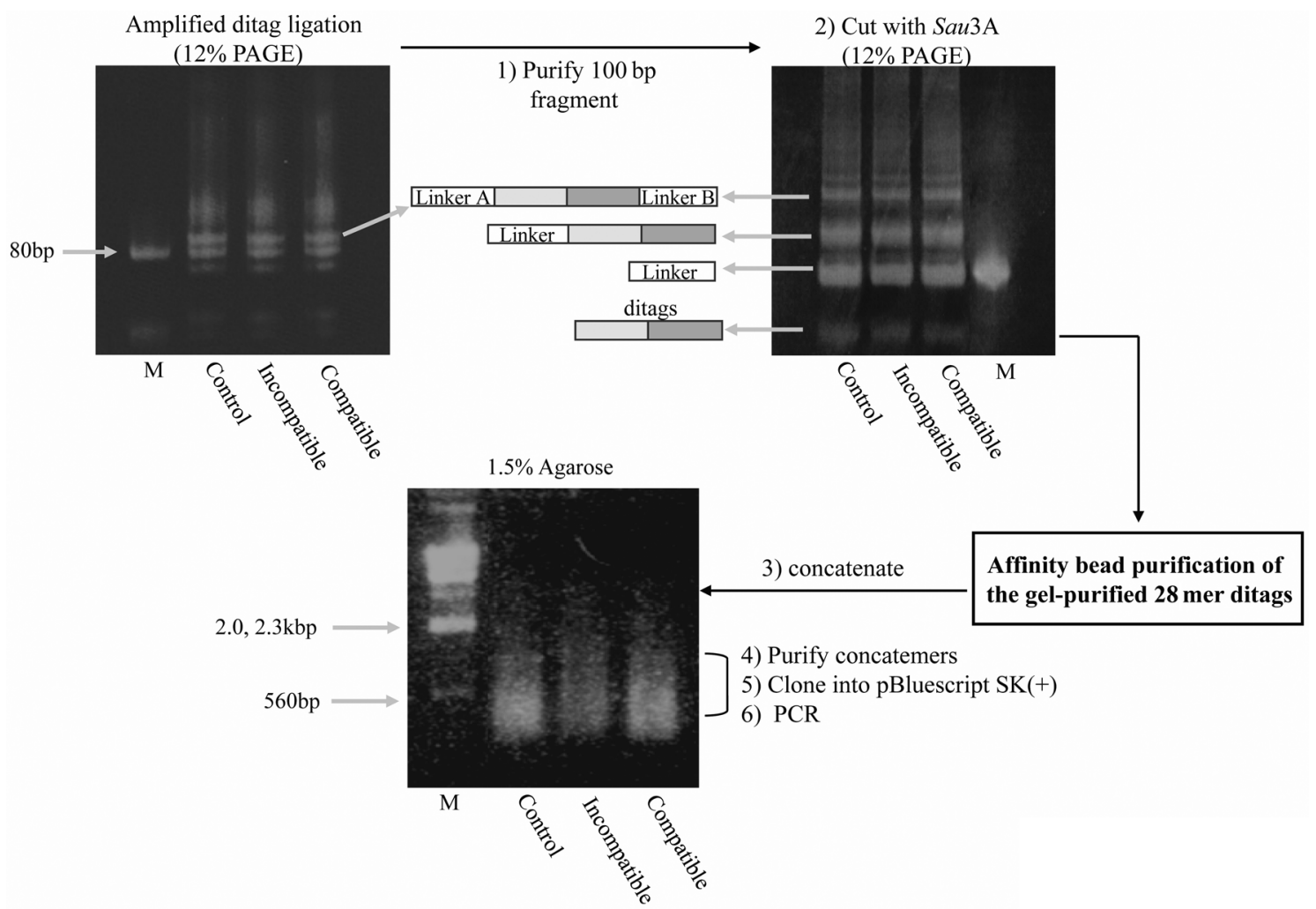

Fig. 2. Steps of the SAGE method repeated for multiple ditags amplifications. Linker-ditag molecules were created through a series of enzymatic steps outlined in the SAGE protocol. Dilutions of substrate were used multiple ditags amplification from the same ditag ligation. Amplified ditags were gel purified (step 1) and digested with Sau3A1 (step 2). Free ditags were gel purified, affinity bead purified, and concatenated (step 3). Large concatemers were gel purified (step 4) and cloned into plasmids (step 5). Individual clones were amplified by PCR (step 6) for sequencing. (M, Marker; C, Control; R, Resistance; S, Susceptible). 
Table 1. The number of expressed genes in different functional categories

\begin{tabular}{lccc}
\hline \hline Functional categories & Control & $\begin{array}{c}\text { Incom- } \\
\text { patible }\end{array}$ & Compatible \\
\hline Development & $1(0.6 \%)$ & $7(1.9 \%)$ & $4(1.1 \%)$ \\
Structure & $14(7.9 \%)$ & $39(10.5 \%)$ & $39(10.8 \%)$ \\
Transcription/translation & $5(2.8 \%)$ & $12(3.2 \%)$ & $15(4.1 \%)$ \\
Stress/defense & $19(11 \%)$ & $59(15.9 \%)$ & $45(12.4 \%)$ \\
Signal & $4(2.3 \%)$ & $17(4.6 \%)$ & $17(4.7 \%)$ \\
Metabolism & $26(14.7 \%)$ & $34(9.1 \%)$ & $38(10.5 \%)$ \\
Photosynthesis & $68(38.6 \%)$ & $118(31.6 \%)$ & $118(32.6 \%)$ \\
Unknown/hypothetical & $13(7.4 \%)$ & $43(11.6 \%)$ & $49(13.5 \%)$ \\
$\quad$ protein & & & \\
Others & $26(14.7 \%)$ & $43(11.6 \%)$ & $37(10.2 \%)$ \\
\hline
\end{tabular}

Bank database. One would assume that SAGE technology would miss the opportunity to find more stress/defense related genes. As the annotation of the rice genome has not been complete, the possibility is always present.

We grouped the gene information into nine functional categories: development, structure, transcription/translation, stress/defense, signal, metabolism, others, photosynthesis, and unknown/hypothetical proteins. We quantified the gene expression under rice blast infection by comparing an incompatible interaction and a compatible interaction (Table 1, Fig. 3). Genes in all categories have been up-regulated as the leaves were treated with the rice blast infection. Overall, the percentage of the expressed genes obtained from the incompatible interaction was very similar to that obtained from the compatible interaction in all categories. As expected, the stress/defense genes were also up-regulated under the infection stress. It should be mentioned that the

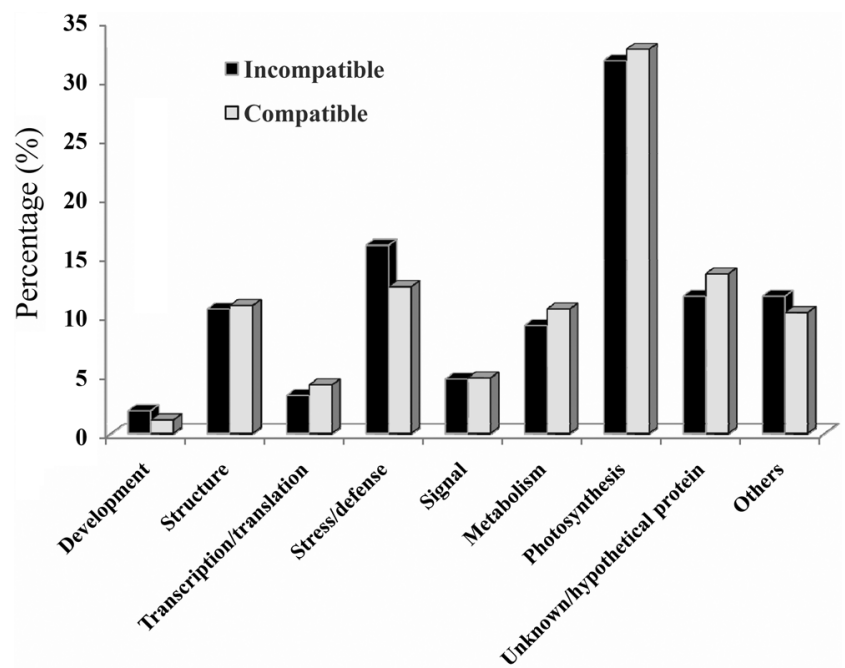

Fig. 3. Functional categories of rice genes identified by SAGE during rice and rice blast fungus interactions. White bars: Compatible interaction. Black bars: Incompatible interaction. increase in the stress/defense gene expression for the incompatible interaction is not conclusively significant compared with that for the compatible interaction. However, there is a noticeable increase in the gene expression level in the incompatible interaction. The pathogen responsive genes isolated from SAGE, beta-glucanase, peroxidases, pathogen-related protein-10 (PR-10), PR-1, Thaumain like protein (TLP), ADP-ribosylation factor, and chitinase are good candidates for genes that are involved in plant defense responses. We summarized the candidate genes that were expressed in the incompatible interaction (Table 2). Several tag sequences were detected multiple times. The SAGE tag for beta-glucanase gene was detected six times, the cases for peroxidase four times, and the putative peroxidase genes twice. In general, both beta-glucanases and peroxidases have been known as enzymes that mediate the defense system against abiotic stresses such as ozone, $\mathrm{H}_{2} \mathrm{O}_{2}$ and ultraviolet radiation (Cheong et al., 2000; Dabir et al., 2007; Kim et al., 2008b; Thalmair et al., 1996). The tags for PR-10 (Kim et al., 2003; McGee et al., 2001; Tanaka et al., 2003) were detected a total of three times, suggesting that the protein seems to make a significant contribution as a biotic stress defense system. Recently, it was reported that the JIOsPR10 protein which is one of the PR-10 family possesses RNase activity that is dependent on sensitive to DTT, suggesting the importance of the disulfide bonding between cysteine residues (Kim et al., 2008a). In addition, expression level using Western blot, immunohistochemical, and its promoter analysis of PBZ1 protein (PR-10 family) is tightly associated with plant cell death tissues, suggesting that PBZ1 could serve as a cell death marker protein as well as defense protein in rice (Kim et al., 2008b). Thus, they might play a role in self-defense mechanisms in plants against biotic and abiotic stresses. However, more studies are needed to understand the biological roles of these differentially expressed proteins.

To more investigate TLP (access number: TC49173), we assessed the TLP gene expression at the mRNA level using Northern blot analysis which was conducted with $20 \mathrm{mg}$ of total mRNA in a lane and hybridized with TLP probe (see Materials and Methods) (Fig. 4A). Total RNA was extracted from the rice leaves inoculated with $M$. grisea along with the control leaves; the intensity of the total RNA stained by ethidium bromide was used as a control. The Northern blot analysis showed that in the incompatible interaction, the level of TLP gene expression was dramatically increased around $12 \mathrm{hrs}$ after the fungal infection while the TLP gene expression level in the compatible interaction was gradually increased as a function of time. We were able to reproduce these results from three independent experimental determinations.

To confirm the protein level expression, we performed a 
Table 2. Expression of pathogen related genes in the incompatible interaction

\begin{tabular}{lclc}
\hline \hline Tag sequence & $\begin{array}{c}\text { Number } \\
\text { of tags }\end{array}$ & \multicolumn{1}{c}{ Corresponding gene } & $\begin{array}{c}\text { Accession no. } \\
\text { in DNA database }\end{array}$ \\
\hline TACAACCAGA & 6 & Beta-glucanase & TC48170 \\
AGGCTCAGCT & 4 & Peroxidase & TC52171 \\
GACGAGCACC & 2 & Putative peroxidase & TC49011 \\
AGTGTAAAAG & 2 & Pathogen-related protein (PR) 10 & TC54328 \\
GAAATAAAGC & 1 & Pathogen-related protein (PR)-10 & TC48653 \\
TTATTATATA & 1 & Pathogen-related protein (PR)-1 & TC52554 \\
GATAATCATC & 1 & Thaumatin-like protein (TLP) & TC49173 \\
ACCGACAAGC & 1 & ADP-ribosylation factor & TC48133 \\
GTATATATAC & 1 & Chitinase & TC49732 \\
\hline
\end{tabular}

Tags are presented as a 10 bp sequence excluding the Sau3A1 (GATC)

A

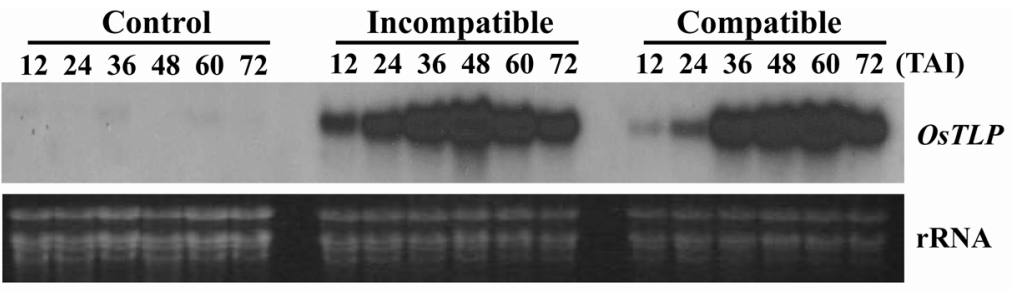

B
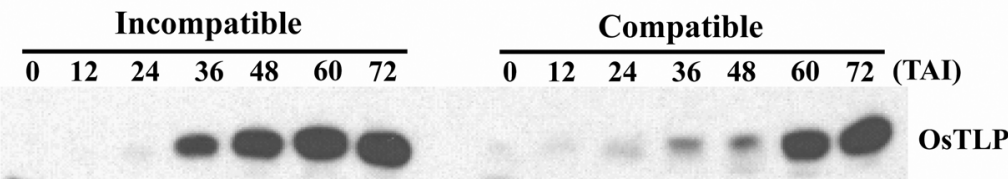

Fig. 4. Time-course accumulation of TLP in response to incompatible and compatible interactions between rice and rice blast fungus. A) Northern blot analysis of cDNA cloned TLP. Rice leaves were inoculated with rice blast fungus of either a race incompatible (KJ401) or compatible (KJ301) to cultivar Jinheung at a concentration of $1 \times 10^{5}$ spores $/ \mathrm{mL}$. Samples were harvested at each time point (12, 24, 36, $48,60,72 \mathrm{~h})$ and total RNA were extracted. Each RNA sample $(20 \mu \mathrm{g})$ was blotted onto a nylon membrane and hybridized with ${ }^{32} \mathrm{P}-$ labeled TLP cDNA probes and washed at high stringency. Equal loading of total RNA was verified by staining rRNA with ethidium bromide. The blots were exposed to X-ray film at $-70^{\circ} \mathrm{C}$ for one day. B) Western blot analysis of TLP from rice leaves. Whole Jinheung rice plants were used to monitor the accumulation of TLP in response to infection with $M$. grisea race KJ401 (incompatible) or race KJ101 (compatible). Each protein sample $(20 \mu \mathrm{g})$ was loaded onto a 12\% SDS-PAGE gel, electrophoresed, and blotted onto a PVDF membrane. Equal loading of protein was confirmed by Ponceau $\mathrm{S}$ staining of the membrane. The membrane was incubated with each purified antibody (dilution, 1:1000) for $2 \mathrm{~h}$. A goat anti-rabbit IgG conjugated with horseradish peroxidase was used as a secondary antibody, and the signal was detected using ECL.

Western blotting analysis which allows us to detect the TLP in the rice tissue extract that had previously been treated with M. grisea (Fig. 4B). We loaded the tissue extract samples ( $20 \mu \mathrm{g}$ per lane) on $12 \%$ SDS polyacrylamide gel, and then transferred the protein onto PVDF membrane, followed by detection using the antibody against the TLP protein. The Western blotting results showed that for the for incompatible interaction, the TLP protein expression was significantly increased 36 hours after the fungal infection, while in the compatible interaction, the protein level gradually increased, but not dramatically. These results were observed with three independent experimental determinations. These results suggest that the early TLP upregulated gene expression in the incompatible interaction helps defend against the infection. In contrast, TLP gene expression in the compatible interaction gradually induced at $60 \mathrm{~h}$ after inoculation appears not to suffice in defending against the fungal infection. However, the functional study of TLP protein remains unknown. Thus further investigation is needed to understand the mechanism and regulation of the TLP protein.

In this study, we identified genes that are up-regulated in response to the fungal $M$. grisea infection stress using SAGE technology. We modified the original SAGE protocol by bead purification for 28 mer ditags, allowing us to increase the yield of restriction endonuclease activity and the formation of concatemers. We also demonstrated that after grouping the genes that are collected by SAGE into nine different categories, the gene expression of the stress/ defense category in the incompatible interaction appears 
particularly up-regulated. We have pointed out that the genes of beta-glucanase, peroxidases, PR-10, PR-1, TLP, ADP-ribosylation factor, and chitinase specifically participate in up-regulation. In particular, we selected TLP, which is directly related to pathogenic infection, finding that timely recognition of the invading pathogen by the resistance gene product in the host triggers rapid and effective defense response. The results obtained using SAGE technology were compared to the data from the proteomics that we previously studied (Kim et al., 2004b). The proteomics investigation showed that two beta-glucanases, TLP, peroxidase, and PR-10 appeared to be up-regulated in the protein expression level in rice under $M$. grisea infection. The agreement in the results from both the transcriptomics (using SAGE technology) and the proteomics strongly suggests that they appear to play a crucial role in rice blast disease. Thus, SAGE technology provided insight into the identities of the genes and their expression levels under rice blast infection, which may lead to development of novel strategies for broad-spectrum and durable disease control.

\section{Acknowledgements}

The authors would like to thank the Baylor University and Gyeongsang National University research exchange program. We also thank Alexander G. Cole and Sara Rae Schlesinger for reviewing this manuscript. This work was supported by a grant from MOST/KOSEF/ to the Environmental Biotechnology National Core Research Center (R15-2003-012-01002-0) and Biogreen 21 (03-2008-0172).

\section{References}

Angelastro, J. M., Klimaschewski, L. P. and Vitolo, O. V. 2000. Improved NlaIII digestion of PAGE-purified $102 \mathrm{bp}$ ditags by addition of a single purification step in both the SAGE and microSAGE protocols. Nucleic Acids Res. 28:E62.

Cheong, Y. H., Kim, C. Y., Chun, H. J., Moon, B. C., Park, H. C., Kim, J. K., Lee, S., Han, C., Lee, S. Y. and Cho, M. J. 2000. Molecular cloning of a soybean class III beta-1,3-glucanase gene that is regulated both developmentally and in response to pathogen infection. Plant Sci. 154:71-81.

Church, G. M. and Gilbert, W. 1984. Genomic sequencing. Proc. Natl. Acad. Sci. USA 81:1991-1995.

Dabir, D. V., Leverich, E. P., Kim, S. K., Tsai, F. D., Hirasawa, M., Knaff, D. B. and Koehler, C. M. 2007. A role for cytochrome $\mathrm{c}$ and cytochrome c peroxidase in electron shuttling from Erv1. EMBO J. 26:4801-4811.

Datson, N. A., van der Perk-de Jong, J., van den Berg, M. P., de Kloet, E. R. and Vreugdenhil, E. 1999. MicroSAGE: a modified procedure for serial analysis of gene expression in limited amounts of tissue. Nucleic Acids Res. 27:1300-1307.

De Jong, J. C., McCormack, B. J., Smirnoff, N. and Talbot, N. J.
1997. Glycerol generates turgor in rice blast. Nature 389:244245.

Hamer, J. E. and Talbot, N. J. 1998. Infection-related development in the rice blast fungus Magnaporthe grisea. Curr. Opin. Microbiol. 1:693-697.

Hamer, J. E., Howard, R. J., Chumley, F. G. and Valent, B. 1988. A Mechanism for Surface Attachment in Spores of a Plant Pathogenic Fungus. Science 239:288-290.

International rice genome sequencing project. 2005. The mapbased sequence of the rice genome. Nature 436:793-800.

Kenzelmann, M. and Muhlemann, K. 1999. Substantially enhanced cloning efficiency of SAGE (Serial Analysis of Gene Expression) by adding a heating step to the original protocol. Nucleic Acids Res. 27:917-918.

Kim, S., Ahn, I. P. and Lee, Y. H. 2001. Analysis of genes expressed during rice-Magnaporthe grisea interactions. Mol. Plant-Microbe Interact. 14:1340-1346.

Kim, S. T., Cho, K. S., Yu, S., Kim, S. G., Hong, J. C., Han, C. D., Bae, D. W., Nam, M. H. and Kang, K. Y. 2003. Proteomic analysis of differentially expressed proteins induced by rice blast fungus and elicitor in suspension-cultured rice cells. Proteomics 3:2368-2378.

Kim, S. T., Kim, S. G., Hwang, D. H., Kang, S. Y., Kim, H. J., Lee, B. H., Lee, J. J. and Kang, K. Y. 2004. Proteomic analysis of pathogen-responsive proteins from rice leaves induced by rice blast fungus, Magnaporthe grisea. Proteomics 4:35693578.

Kim, S. T., Yu, S., Kang, Y. H., Kim, S. G., Kim, J. Y., Kim, S. H. and Kang, K. Y. 2008a. The rice pathogen-related protein 10 (JIOsPR10) is induced by abiotic and biotic stresses and exhibits ribonuclease activity. Plant Cell Rep. 27:593-603.

Kim, S. T., Kim, S. G., Kang, Y. H., Wang, Y., Kim, J.-Y., Yi, N., Kim, J. K., Rakwal, R., Koh, H. J. and Kang, K. Y. 2008b. Proteomics analysis of rice lesion mimic mutant (spl1) reveals tightly localized probenazole-induced protein (PBZ1) in cells undergoing programmed cell death. J. Proteome Res. 7:17501760.

Madden, S. L., Wang, C. J. and Landes, G. 2000. Serial analysis of gene expression: from gene discovery to target identification. Drug Discov Today 5:415-425.

Margulies, E. H., Kardia, S. L. and Innis, J. W. 2001. Identification and prevention of a GC content bias in SAGE libraries. Nucleic Acids Res. 29:E60-60.

McGee, J. D., Hamer, J. E. and Hodges, T. K. 2001. Characterization of a PR-10 pathogenesis-related gene family induced in rice during infection with Magnaporthe grisea. Mol. PlantMicrobe Interact. 14:877-886.

Munasinghe, A., Patankar, S., Cook, B. P., Madden, S. L., Martin, R. K., Kyle, D. E., Shoaibi, A., Cummings, L. M. and Wirth, D. F. 2001. Serial analysis of gene expression (SAGE) in Plasmodium falciparum: application of the technique to A-T rich genomes. Mol. Biochem. Parasitol. 113:23-34.

Peters, D. G., Kassam, A. B., Yonas, H., O'Hare, E. H., Ferrell, R. E. and Brufsky, A. M. 1999. Comprehensive transcript analysis in small quantities of mRNA by SAGE-lite. Nucleic Acids Res. 27:e39. 
Powell, J. 1998. Enhanced concatemer cloning-a modification to the SAGE (Serial Analysis of Gene Expression) technique. Nucleic Acids Res. 26:3445-3446.

Talbot, N. J. 2003. On the trail of a cereal killer: Exploring the biology of Magnaporthe grisea. Annu. Rev. Microbiol. 57:177202.

Tanaka, N., Che, F. S., Watanabe, N., Fujiwara, S., Takayama, S. and Isogai, A. 2003. Flagellin from an incompatible strain of Acidovorax avenae mediates $\mathrm{H}_{2} \mathrm{O}_{2}$ generation accompanying hypersensitive cell death and expression of PAL, Cht-1, and PBZ1, but not of Lox in rice. Mol. Plant-Microbe Interact. 16:422-428.

Thalmair, M., Bauw, G., Thiel, S., Dohring, T., Langebartels, C. and Sandermann, H. 1996. Ozone and ultraviolet B effects on the defense-related proteins $\beta$-1,3-glucanase and chitinase in tobacco. J. Plant Physiol. 148:222-228.

Valent, B. and Chumley, F. G. 1991. Molecular genetic analysis of the rice blast fungus, Magnaporthe grisea. Annu. Rev. Phytopathol. 29:443-457.

van den Berg, A., van der Leij, J. and Poppema, S. 1999. Serial analysis of gene expression: rapid RT-PCR analysis of unknown SAGE tags. Nucleic Acids Res. 27:e17.

Velculescu, V. E., Zhang, L., Vogelstein, B. and Kinzler, K. W. 1995. Serial analysis of gene expression. Science 270:484487.

Yamamoto, M., Wakatsuki, T., Hada, A. and Ryo, A. 2001. Use of serial analysis of gene expression (SAGE) technology. $J$. Immunol. Methods 250:45-66.

Zeifler, R. S., Leong, S. A. and Teeng, P. S. 1994. Rice Blast Disease. CAB International, Wallingford. 